\title{
A Numerical Investigation of the Performance of Linear Interpolation Schemes Coupled Finite Volume Method in the Analysis of Confined Convection- Diffusion Turbulent Flow Field
}

\author{
Jane Gatwiri, Stephen Karanja, David Theuri
}

\begin{abstract}
Numerical solutions are never exact due to errors emanating from the scheme used in discretizing the governing equations and the flow domain. For convection-diffusion flow, the magnitude of these errors varies depending on the scheme used to interpolate the nodal values of the flow quantities to the interfaces. An interpolation scheme that minimizes these errors would give results that are consistent to experimental results. This paper documents the performance of three linear interpolation schemes; upwind differencing, central differencing scheme and the hybrid scheme in obtaining temperature profiles for a convection-diffusion turbulent flow field. To eliminate the enormous scales inherent in turbulent flow, the field variables present in the governing equations are decomposed into a mean and a fluctuating component and averaged. The closure problem was solved using the $S S T-k-\omega$ turbulence model. The resulting equations are discretized using the robust finite volume discretization technique. The discretized equations are solved using a segregated pressure-based algorithm. The results revealed that the central difference interpolation scheme generate temperature profiles that were consistent with experimental results of Ampofo and Karayiannis, (2003).
\end{abstract}

Keywords - Convetion, diffusion, discretization, interpolation;turbulence.

\section{BACKGROUND}

In fluid dynamics, flow behavior is analyzed experimentally, analytically or numerically. Though experimental methods are capable of producing physically realistic results, they are expensive, and less flexible. Analytical solutions of most flows occurring in practical applications are either unavailable, or computationally inefficient. Solutions of most flows are thus obtained numerically. However, the results are never exact due to discretization errors inherent in numerical methods. In the Finite volume discretization method, the solution domain is subdivided into finite volumes. The transported variable is stored at the center of the finite volume. Since the flow is continuous, the flux entering a given volume is identical to the flux leaving the adjacent volume then, the method is conservative. The finite volume discretization method is geometrically flexible and can therefore be used for any type of geometry. The method uses integral formulation of conservation laws which makes it superior to other numerical methods because in differential form, one makes the assumption that the solution is continuous. The integral formulation avoids the assumption of smoothness. Nevertheless, one major challenge is obtaining the interface values of flow variables in a convection-diffusion flow field. Various schemes that interpolate the centered values of the flow quantities to the interfaces are found in literature. Each of these schemes is anchored on a certain assumption about the distribution of the flow quantity from the center of a finite volume to the interfaces. This paper documents the performance of three linear interpolation schemes in analyzing temperature profiles for a convection-diffusion turbulent flow in a cavity.

\section{A. Review of Previous Related Studies}

Numerical methods have over the years, been used to produce quantitative predictions of fluid flow phenomena based on the conservation laws that govern fluid flow. A comparative study of three turbulence models in predicting heat transfer due to natural convection inside an air filled cavity was done by Awuor in 2013. The cavity was maintained at a temperature of $283 \mathrm{k}$ on the cold wall and 323k on the opposite hot wall. The numerical method used was the Finite Difference Approximation, coupled with the Hybrid differencing scheme. The results showed that the $k-\omega-S S T$ model performed better than the $k-\varepsilon$ and the $k-\omega$ turbulence models in the whole enclosure [1].

Published on November 30, 2021.

J. Gatwiri, Department of Mathematics, Meru University of Science and Technology, Meru, Kenya.

(corresponding e-mail: janemuteagatwiri@gmail.com)

S. Karanja, Department of Mathematics, Meru University of Science and Technology, Meru Kenya.

(e-mail: stephenkaranja16@gmail.com)

D. Theuri, Department of Pure and Applied Mathematics, Jomo Kenyatta University of Agriculture and Technology, Kenya.

(e-mail: dtheuri@gmail.com) 
Reference [2] studied natural convection of air in a square cavity with partially thermally active side walls. The behavior of boundary layers at variable Rayleigh numbers was analyzed. The Finite Volume Method was used to discretize the governing equations. The Central Differencing interpolation scheme was used to obtain interface values. Results showed that for $10^{3} \leq R a \leq 10^{6}$, the flow regime was laminar.

In 2015, numerical analysis of a steady natural convection phenomena of air in a square cavity heated from the bottom was carried out. The governing equations along with the boundary conditions were discretized using the Finite Difference Method coupled with the central difference interpolation scheme. Results showed that increase in temperature difference between the vertical walls affects the fluid dynamic behavior by increasing the intensity of flow in the enclosure [3].

The above researchers have used a particular interpolation scheme without an apparent justification. This study endeavors to establish a linear interpolation scheme that can be coupled with the Finite Volume discretization method to generate temperature profiles that are consistent with experimental results.

\section{Governing Equations}

The flow is governed by the conservation laws of motion coupled with Boussinesq approximation. The field variables are decomposed into a mean and a fluctuating and averaged. The following assumptions apply in this study.

- $\quad$ The fluid is Newtonian and Boussinesq.

- The flow regime is buoyancy driven and turbulent.

- Gravity is the only body force acting on the fluid.

- Effects caused by viscous dissipations are neglected.

The general equations governing the fluid flow include:

- Mass Conservation Equation

$$
\frac{\partial \rho}{\partial t}+\frac{\partial}{\partial x_{j}}\left(\rho u_{j}\right)=0
$$

- Momentum Conservation Equation

$$
\frac{\partial}{\partial t}\left(\rho u_{i}\right)+\frac{\partial}{\partial x_{j}}\left(\rho u_{i} u_{j}\right)=-\frac{\partial P}{\partial x_{i}}+\rho g_{i}+\frac{\partial}{\partial x_{j}}\left[\mu\left(\frac{\partial u_{i}}{\partial x_{j}}+\frac{\partial u_{j}}{\partial x_{i}}\right)+\mu_{s} \delta_{i j} \frac{\partial u_{k}}{\partial x_{k}}\right]
$$

- Energy Conservation Equation

$$
\rho\left[\frac{\partial}{\partial t}\left(C_{P} T\right)+\frac{\partial}{\partial x_{j}}\left(C_{p} u_{j} T\right)\right]=\frac{\partial}{\partial x_{j}}\left(\lambda \frac{\partial T}{\partial x_{j}}\right)+\beta T\left(\frac{\partial p}{\partial t}+\frac{\partial u_{j} p}{\partial x_{j}}\right)+\Phi
$$

In a turbulent flow field, each of the flow variables is regarded as consisting of a mean or average value $(\phi)$ and a fluctuating value $\left(\phi^{\prime}\right)$ such that: $\phi(x, t)=\phi^{\prime}(x, t)+\bar{\phi}(t)$ (4) $\phi$ Denotes the value of a flow property at a particular instance and location, whereas $\bar{\phi}$ and $\phi^{\prime}$ denotes the mean and fluctuating values of $\phi$ respectively. This technique of separating the average and the fluctuating parts of a quantity is referred to as Reynolds decomposition. The average value $\bar{\phi}$ is defined as:

$$
\bar{\phi}=\frac{1}{\Delta t} \int_{t_{0}}^{t_{0}+\Delta t} \phi(x, t) d t
$$

where $\Delta t$ is the time averaging interval chosen such that it is sufficiently large. This implies that the mean value $\bar{\phi}$ is a function of space only. Consequently,

$$
\bar{\phi}(x, t)=\bar{\phi}\left(x_{i}\right)(6)
$$


The governing (1)-(3) are decomposed and averaged, and assumptions coupled with the Boussinesq assumption imposed to yield:

$$
\begin{gathered}
\frac{\partial \overline{U_{j}}}{\partial x_{j}}=0 \\
\frac{\partial U_{i}}{\partial t}+U_{j} \frac{\partial U_{i}}{\partial x_{j}}=-\frac{1}{\rho} \frac{\partial P}{\partial x_{i}}+\frac{\partial}{\partial x_{j}}\left[v \frac{\partial U_{i}}{\partial x_{j}}-\overline{u_{i} u_{j}}\right]+\left[1-\beta\left(T-T_{0}\right)\right] g_{i} \\
\left.\frac{\partial T}{\partial t}+U_{j} \frac{\partial T}{\partial x_{j}}=\alpha \frac{\partial^{2} T}{\partial x_{j}^{2}}-\frac{\partial}{\partial x_{j}} \overline{\left(u_{j} T\right.}\right)(9)
\end{gathered}
$$

\section{A. Treatment of Turbulent Correlations}

In (8) and (9), the correlations $\overline{u_{i} u_{j}}$ and $\overline{u_{j} T}$ are unknown. These terms emanate from the fluctuation motion. In addition to the stresses caused by pressure and viscosity effects, a fluid element experiences stresses emanating from the turbulent fluctuations. These correlations account for the additional momentum and energy transport in turbulent flow. The presence of these turbulent correlations in the Reynolds averaged equations therefore creates need for closure. Researchers have over the years attempted to resolve the closure problem. Among the numerous attempts is turbulence modeling, an approach that either expresses the turbulent correlations in terms of known quantities or through direct resolution of the correlations. In turbulence modeling, the $k$-equation is the most fundamental since it describes the budget of turbulent kinetic energy. The equation is given as

$$
\left\{\begin{array}{l}
\underbrace{\frac{\partial}{\partial t}(\overline{\rho k})}_{I}+\underbrace{\frac{\partial}{\partial x_{j}}\left(\overline{\rho u_{j} k}\right)}_{I I}=-\frac{\partial}{\partial x_{j}}(\underbrace{\frac{1}{2 \overline{\rho u_{i}^{\prime} u_{i}^{\prime} u_{j}^{\prime}}}+\underbrace{\overline{p^{\prime} u_{i}^{\prime}}}_{I V}-\underbrace{\mu \frac{\partial k}{\partial x_{j}}}_{V})-\underbrace{\overline{\rho u_{i}^{\prime} u_{j}^{\prime}} \frac{\partial u_{i}}{\partial x_{j}}}_{V I}}_{I I I} \\
+\underbrace{\beta g \rho \overline{u_{i}^{\prime} T^{\prime}}}_{V I I}-\underbrace{\mu \frac{\partial u_{i}^{\prime}}{\partial x_{j}} \frac{\partial u_{i}^{\prime}}{\partial x_{j}}}_{V I I I}
\end{array}\right\}
$$

The turbulent dissipation $\varepsilon$ in the $k-\varepsilon$ model represents the rate of dissipation of turbulent kinetic energy. The $\varepsilon$ - equation is derived from the Navier- Stokes equation for fluctuation vorticity.

\section{B. Modeling the Unknown Correlations in the $k$-Equation}

The kinetic energy (10) has most of its terms containing unknown correlations emanating from velocity fluctuations in the turbulent flow. It is therefore necessary that we model these correlations by use of similarity considerations together with the Boussinesq assumptions. The modeled kinetic energy equation is given as:

$$
\frac{\partial k}{\partial t}+U_{j} \frac{\partial k}{\partial x_{j}}=v_{t}\left[\frac{\partial u_{i}}{\partial x_{j}}+\frac{\partial u_{j}}{\partial x_{i}}\right] \frac{\partial u_{i}}{\partial x_{j}}+\frac{\partial}{\partial x_{j}}\left[\left(v \sigma_{k}+v_{t}\right) \frac{\partial k}{\partial x_{j}}\right]+\beta g \frac{v_{t}}{\sigma_{k}} \frac{\partial T}{\partial x_{i}}-\varepsilon(11)
$$

Where $\varepsilon$ is the dissipation rate of turbulent kinetic energy.

$$
k-\varepsilon \quad \varepsilon
$$

In order to obtain the turbulence model, we need an equation for expressed as follows:

$$
\varepsilon=c_{\mu} k \omega
$$

Substituting (12) into (11) yield: 


$$
\left\{\begin{array}{l}
\frac{\partial k}{\partial t}+U_{j} \frac{\partial k}{\partial x_{j}}=v_{t}\left[\frac{\partial u_{i}}{\partial x_{j}}+\frac{\partial u_{j}}{\partial x_{i}}\right] \frac{\partial u_{i}}{\partial x_{j}}+\frac{\partial}{\partial x_{j}}\left[\left(v \sigma_{k}+v_{t}\right) \frac{\partial k}{\partial x_{j}}\right] \\
+\beta g \frac{v_{t}}{\sigma_{k}} \frac{\partial T}{\partial x_{i}}-c_{\mu} k \omega
\end{array}\right\}
$$

\section{The Shear-Stress Transport Turbulence Model}

The $k-\varepsilon$ turbulence model is preferred in explaining flow behavior in the free stream region of a flow domain, while the $k-\omega$ model best accounts for flow behavior near a solid boundary [7] developed the SST $k-\omega$ turbulence model by putting into consideration the strengths and weaknesses of the $k-\varepsilon$ and the $k-\omega$ turbulence models. This model makes use of the $k-\omega$ model near the solid boundary, and transforms to the $k-\varepsilon$ model in the free stream region. For this reason, SST $k-\omega$ the turbulence model is considered in the current study because it well accounts for the flow behavior in the entire flow domain.

The turbulence equations for the SST $k-\omega$ are represented as follows:

$$
\begin{array}{r}
\frac{\partial k}{\partial t}+U_{j} \frac{\partial k}{\partial x_{j}}=\frac{\partial}{\partial x_{j}}\left(\Gamma_{k} \frac{\partial k}{\partial x_{j}}\right)+P_{k}+G_{k}-Y_{k} \\
\frac{\partial \omega}{\partial t}+U_{j} \frac{\partial \omega}{\partial x_{j}}=\frac{\partial}{\partial x_{j}}\left(\Gamma_{\omega} \frac{\partial \omega}{\partial x_{j}}\right)+P_{\omega}+D \omega-Y_{\omega}
\end{array}
$$

The modeled turbulent kinetic energy equation is represented by (14). Using a similar approach, we now model (15) as follows:

$$
\left\{\begin{array}{l}
\Gamma_{\omega}=\left(v+\sigma_{\omega} v_{t}\right), P_{\omega}=\gamma P_{k}, Y_{\omega}=\beta^{*} \omega^{2} \\
D_{\omega}=2 \sigma \omega_{2}\left(1-F_{1}\right) \frac{1}{\omega} \frac{\partial k}{\partial x_{j}} \frac{\partial \omega}{\partial x_{j}}
\end{array}\right\}
$$

Substituting (16) into (15) yield:

$$
\left\{\begin{array}{l}
\frac{\partial_{\omega}}{\partial t}+U_{j} \frac{\partial \omega}{\partial x_{j}}=\frac{\partial}{\partial x_{j}}\left[\left(v+\sigma_{\omega} v_{t}\right) \frac{\partial \omega}{\partial x_{j}}\right]+\gamma P_{k}+ \\
2 \sigma \omega_{2}\left(1-F_{1}\right) \frac{1}{\omega} \frac{\partial k}{\partial x_{j}} \frac{\partial \omega}{\partial x_{j}}-\beta^{*} \omega^{2}
\end{array}\right\}
$$

(8), (9),(10) (14) and (17) give the final set of model equations.

TABLE I: MOdel CONSTANTS FOR THE SST $k-\omega$ TURBULENCE MOdel

\begin{tabular}{ccccccccccc}
\hline \hline$\sigma_{k, 1}$ & $\sigma_{k, 2}$ & $\sigma_{\omega, 1}$ & $\sigma_{\omega, 2}$ & $\alpha_{*}$ & $c_{\mu}$ & $\beta_{i, 1}$ & $\beta_{i, 2}$ & $\beta_{\infty}^{*}$ & $\beta_{i}$ & $\operatorname{Re}_{\omega}$ \\
\hline \hline 0.85 & 1 & 0.5 & 0.857 & 0.31 & 0.09 & 0.075 & 0.0828 & 0.09 & 0.072 & 2.95 \\
\hline \hline
\end{tabular}

\section{NON-DimensionalizATION OF THE EQUATIONS}

The set of equations governing the flow contain more variables than the number of equations. In fluid dynamics, the use of a suitable non-dimensional scheme is of paramount importance in reducing the number of parameters. In addition, Non dimensionalization enables both experimental and analytical results to be expressed in the most efficient form and makes the solution bounded. Besides, it enables us to obtain results of a flow regime experiencing a given set of conditions by making use of the results of a geometrically similar flow. In this regard, choose $U$ to represent characteristic velocity, $L_{R}$ to represent the characteristic length and $\Delta T_{*}$ to represent the characteristic temperature. All other physical properties will be nondimensionalized using their respective values at a reference temperature $T_{0}$. We now apply the relevant scaling variables to the final set of modeled equations to obtain the non-dimensional form of the turbulent equations as follows: 


$$
\begin{aligned}
& \frac{\partial U_{j}^{\prime}}{\partial X_{j}^{\prime}}=0 \\
& {\left[\begin{array}{l}
\frac{\partial U_{i}^{\prime}}{\partial t^{\prime}}+U_{j}^{\prime} \frac{\partial U_{i}^{\prime}}{\partial X_{j}^{\prime}}=-\frac{1}{\rho^{\prime}} \frac{\partial P^{\prime}}{\partial X_{i}^{\prime}}+\frac{\partial}{\partial X_{j}^{\prime}}\left[v^{\prime} \sqrt{\frac{\operatorname{Pr}}{R a}} \frac{\partial U_{i}^{\prime}}{\partial X_{J}^{\prime}}-\overline{u_{i}^{\prime} u_{j}^{\prime}}\right] \\
+\left[1-\beta^{\prime} \beta_{0}\left(\partial \theta \nabla T_{*}\right)\right] g_{i}^{\prime} g_{R}
\end{array}\right.} \\
& \frac{\partial \theta}{\partial t^{\prime}}+\frac{\partial \theta}{\partial X_{j}^{\prime}}\left[U_{j}^{\prime}-\overline{u_{j}^{\prime}}\right]=\frac{\alpha^{\prime}}{\sqrt{R a \operatorname{Pr}}} \frac{\partial}{\partial X_{j}^{\prime}}\left[\frac{\partial \theta}{\partial X_{j}^{\prime}}\right] \\
& \left\{\begin{array}{l}
\frac{\partial k^{\prime}}{\partial t^{\prime}}+U_{j} \frac{\partial k^{\prime}}{\partial X_{j}^{\prime}}=\frac{v_{t}^{\prime} v_{0}}{L_{R}}\left[\frac{\partial U_{i}^{\prime}}{\partial X_{j}^{\prime}}+\frac{\partial U_{j}^{\prime}}{\partial X_{i}^{\prime}}\right] \frac{\partial U_{i}^{\prime}}{\partial X_{j}^{\prime}}+\frac{\operatorname{Pr}}{R a} \frac{\partial}{\partial X_{j}^{\prime}}\left\{\left(v^{\prime}+v_{t}^{\prime} \sigma_{k}\right) \frac{\partial k}{\partial X_{j}^{\prime}}\right\} \\
+\frac{\beta^{\prime} \beta_{0} g^{\prime} g_{0} v_{t}^{\prime} v_{0} \Delta T_{*} L_{R}^{2}}{\alpha_{0}{ }^{2} R a \operatorname{Pr}}-\frac{\alpha_{0}}{L_{R}} C_{\mu} k^{\prime} \omega^{\prime} \sqrt{R a \operatorname{Pr}}
\end{array}\right\} \\
& \left\{\begin{array}{l}
\frac{\partial \omega^{\prime}}{\partial t^{\prime}}+U_{j}^{\prime} \frac{\partial \omega^{\prime}}{\partial X_{j}^{\prime}}+\frac{\operatorname{Pr}}{R a} \frac{\partial}{\partial X_{j}^{\prime}}\left[\left(v^{\prime}+\sigma_{\omega} v_{t}^{\prime}\right) \frac{\partial \omega^{\prime}}{\partial X_{j}^{\prime}}\right]+\frac{L_{R}}{\alpha_{0}} \frac{\gamma}{\sqrt{R a \operatorname{Pr}}}\left[\frac{\partial U_{i}^{\prime}}{\partial X_{j}^{\prime}}+\frac{\partial U_{j}^{\prime}}{\partial X_{i}^{\prime}}\right] \frac{\partial U_{i}^{\prime}}{\partial X_{j}^{\prime}} \\
-\frac{\alpha_{0}}{L_{R}} \sqrt{R a \operatorname{Pr}} \beta^{*} \omega^{\prime 2}+2 \sigma \omega_{2}\left[1-F_{1}\right] \frac{1}{\alpha_{0} \sqrt{R a \operatorname{Pr}}} \frac{1}{\omega^{\prime}} \frac{\partial k^{\prime}}{\partial X_{j}^{\prime}} \frac{\partial \omega^{\prime}}{\partial X_{j}^{\prime}}
\end{array}\right\}
\end{aligned}
$$

\section{A. Temperature Boundary Conditions}

The non-dimensional temperature is defined as $\theta=\frac{T-T_{0}}{\Delta T_{*}}$ where $\Delta T_{*}$ is the characteristic temperature difference between the hot and cold walls. $\theta$ is conveniently chosen so that $0 \leq \theta \leq 1$ within the cavity. For the adiabatic surfaces of the cavity, $\theta$ is constant. This implies that on these surfaces:

$$
\frac{\partial \theta}{\partial n}=0
$$

where $\mathrm{n}$ is a scalar of the outward unit vector normal to the surface.

In the current study, the two opposite vertical walls of the cavity are differentially heated. Dirichlet boundary conditions are thus applied on the vertical walls so that

$$
\left\{\begin{array}{l}
\theta_{h}=1 \\
\theta_{c}=0
\end{array}\right\}
$$

\section{NUMERICAL METHOD}

The transport equations presented in section 3.0 can be expressed into a generic conservation form that comprises of all the processes that influence the change of a dependent variable in a finite volume. The generic conservation form of the transport equations is given as:

$$
\frac{\partial \phi}{\partial t}+\frac{\partial}{\partial x_{j}}\left(u_{j} \phi\right)=\frac{\partial}{\partial x_{j}}\left(\Gamma \phi \frac{\partial \phi}{\partial x_{j}}\right)+S \phi
$$


Where $\phi, \Gamma_{\phi}$ and $S \phi$ are the dependent variable, exchange coefficient and source term of $\phi$ respectively. The generic conservation equation has four distinct terms which define the processes that affect the transport of $\phi$ in a finite volume. These are the temporal term, the convection term, the diffusion term and the source term. The integral form of the generic conservation equation is given as [8].

$$
\int_{V P} \frac{\partial \phi}{\partial t} d v+\frac{\partial}{\partial x_{j}} \int_{V P}\left(u_{j} \phi\right) d v=\frac{\partial}{\partial x_{j}} \int_{V P}\left(\Gamma_{\phi} \frac{\partial \phi}{\partial x_{j}}\right) d v+\int_{V P} S \phi d v
$$

Both the convection and the diffusion terms of (26) represent the flux of $\phi$ across the faces of a finite volume.

The discretized form of the generic conservation equation is given in summation form as

$$
\phi_{i j}=\frac{\sum a_{n b} \phi_{n b}+b_{i j}}{a_{i j}}
$$

In (27), the coefficient $a_{n b}$ represents the contribution of each of the neighboring finite volumes to the dependent variable $\phi_{i j}$, whereas $a_{i j}$ contains the contribution of all terms influencing $\phi_{i j}$. The coefficient $b$ contains the contribution of the source terms. We obtain a discretization equation of the form of (27) for temperature in each finite volume. We adopt an iterative segregated algorithm to solve the equations. The relation below is used to modify the values of the coefficients obtained in each iteration.

$$
\phi_{i j}=\sigma \phi_{i j}^{\text {predicted }}+(1-\sigma) \phi_{i j}^{o}
$$

the relaxation factor is $\sigma \leq 1$.

In (28), $\phi_{i j}^{\text {predicted }}$ is the value of the new iteration whereas $\phi_{i j}^{o}$ is the value of the solution variable in the previous iteration.

\section{A. Convergence Criterion}

Once the values $\phi_{i j}$ have been obtained for temperature in all the finite volumes, the solution is modified using (28). We now obtain the absolute residual measure of the dependent variable using the relation below:

$$
R_{i j}=\left|a_{i j} \phi_{i j}-\sum_{n b} a_{n b} \phi_{n b}-b\right|
$$

However, since the values of coefficients $a_{n b}$ and $b$ changes in each finite volume, it is necessary that we obtain the local relative error by scaling the local residual relative to local value of the quantity such that

$$
R_{i j}^{\text {scaled }}=\frac{\left|a_{i j} \phi_{i j}-\sum_{n b} a_{n b} \phi_{n b}-b\right|}{a_{i j} \phi_{i j}}
$$

In the entire computational domain, we thus obtain the overall measure of the scaled residual as:

$$
R^{\phi}=\frac{\sum_{A L L C E L L S}\left|a \phi-\sum_{n b} a_{n b} \phi_{n b}-b\right|}{\sum_{A L L C E L L S}|a \phi|}
$$


(31) is used to monitor the convergence of an equation. If the overall residual reduces by three orders of magnitude then, the solution is said to be approaching the actual solution. Once the conservation equations have converged, the solution does not change with further iteration. Consequently, the conservation equations are satisfied in the entire solution domain.

\section{B. Temperature Distribution Across the Faces of the Control Volumes}

The generic conservation equation is imposed over each control volume in the discretized solution domain. A set of discrete equations is obtained for each dependent variable over all the control volumes. To estimate the temperature distribution across the boundaries of the control volumes, three linear interpolation schemes; the central differencing, the upwind differencing and the hybrid schemes were used. Each of the interpolation schemes is anchored on a certain assumption about the distribution of the transported quantity across the boundaries of a control volume. The central difference scheme assumes that the value of the transported variable at an interface is the mean of the values the variable on either side of the face. The face is assumed to be midway between the two nearest nodal points. The upwind difference assumes that the value of the transported quantity at a given face is equal to the value of the variable at the nodal point that is on the upwind side of the face. The hybrid scheme makes use of both the central difference and the upwind schemes for different values of Peclet number. Temperature profiles obtained using each of the interpolation schemes are presented. The profiles have been compared with the experimental results of Ampofo and Karayiannis.

\section{Results AND Discussion}

Results obtained using all the three linear interpolation schemes indicate high temperature gradients along the vertical walls. This is attributed to the convectional currents experienced along the hot walls. Air around the vertical walls gets heated and becomes less dense. Buoyancy force pushes the less dense air upwards and it is replaced by cooler denser air from the top. This generates convectional currents hence the sharp temperature gradients. This is in agreement with the benchmark results. There is a variance between the alignment of the temperature profiles obtained using the upwind ad hybrid schemes when compared with the benchmark results.

However, results obtained using the central difference schemes are in agreement with the experimental results. The alignment of the temperature contours in the central difference results largely match the alignment of the temperature contours experimentally generated. In both results, there are high temperature gradients along the vertical walls with the contours straightening in the interior of the flow domain. This signifies heat transfer by convection along the vertical walls and heat transfer by conduction in the interior of the flow domain.

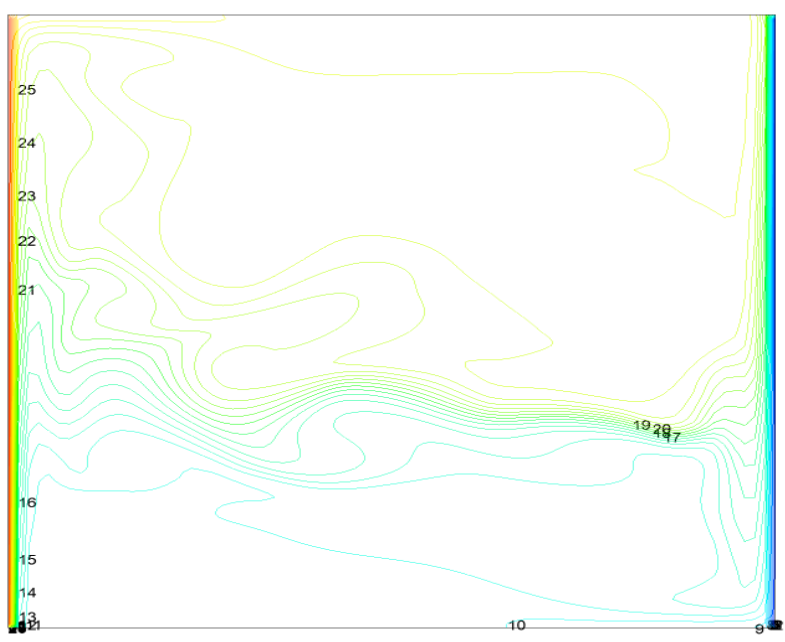

Hybrid Scheme

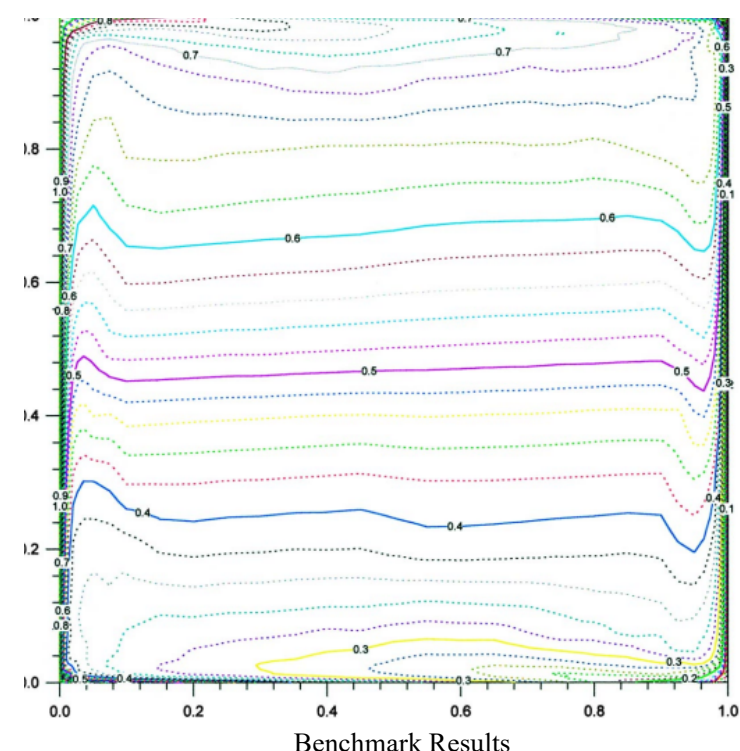



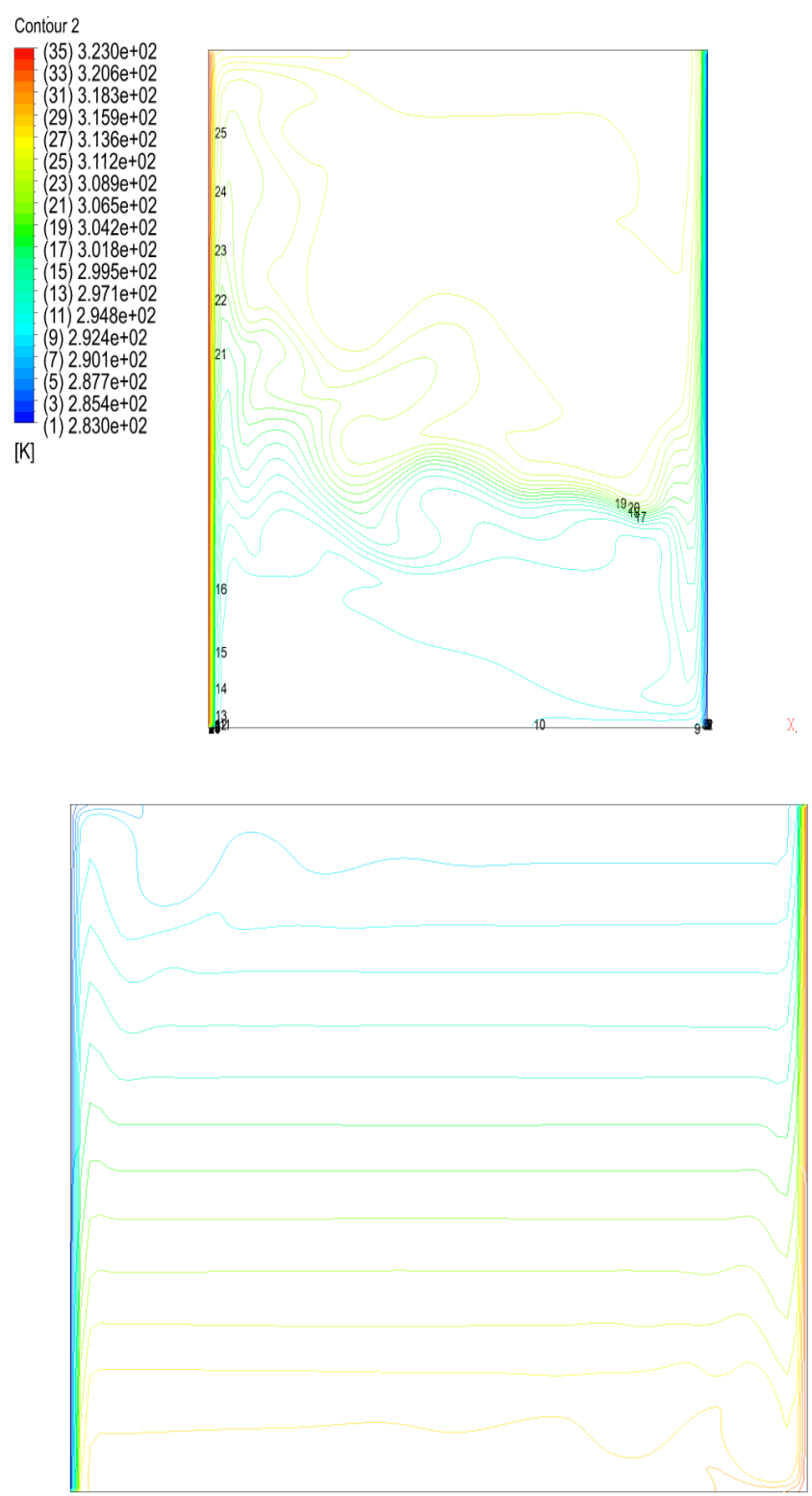

Central Difference Scheme
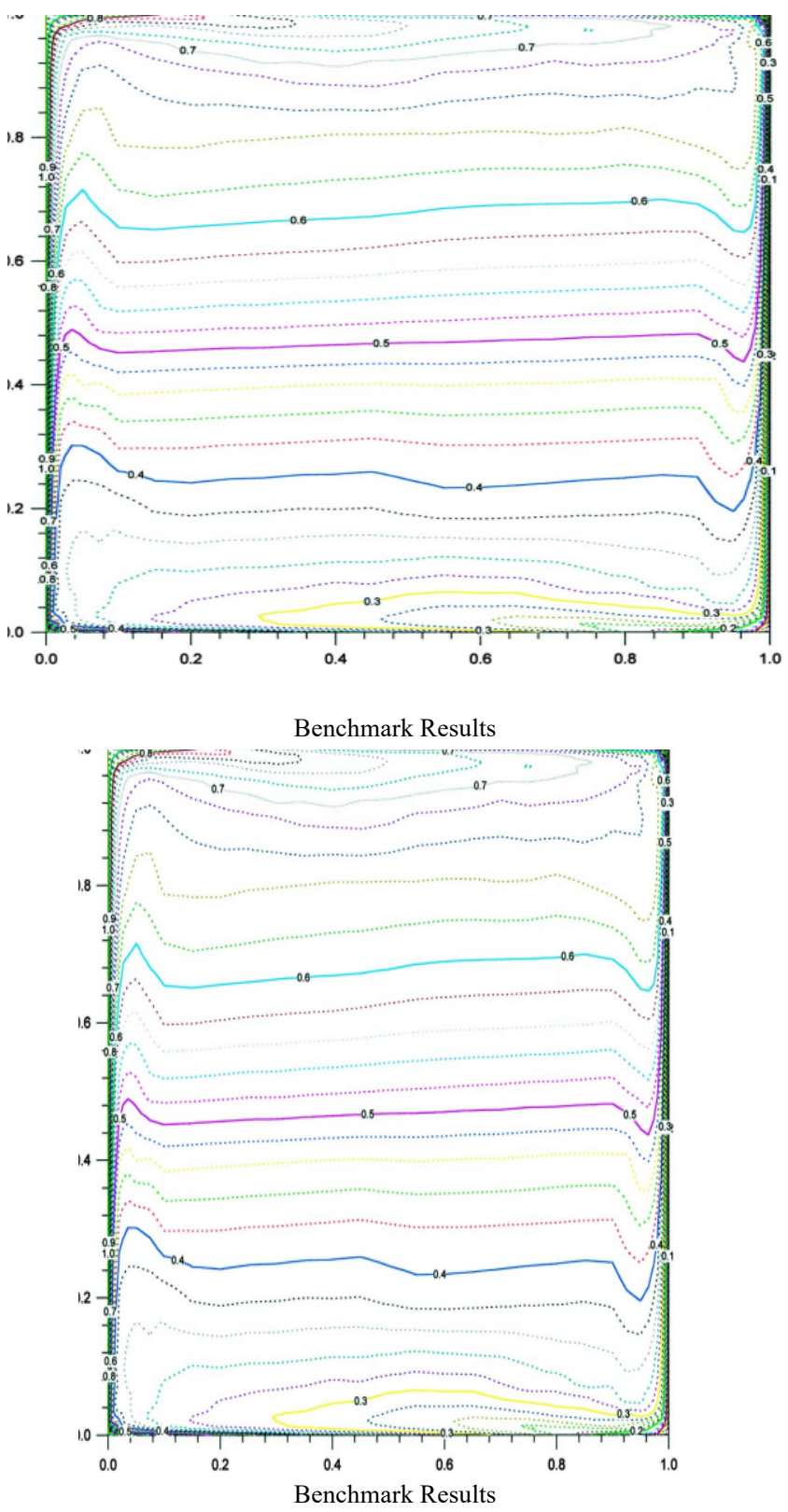

Fig. 1. Comparison Between Numerical Results And Benchmark Results.

\section{CONCLUSION}

Based on the above findings, we conclude that the central difference interpolation scheme is the recommended linear interpolation scheme for analyzing temperature profiles for confined convection-diffusion turbulent flows using the finite volume discretization method.

\section{RECOMMENDATIONS FOR FURTHER STUDY}

In order to gain more acumen on interpolation schemes, we recommend further research in the following areas:

1. Analyzing the distribution of other flow variables like pressure and turbulence in confined convection-diffusion turbulent flows.

2. Extension of the study to include non-linear interpolation schemes like the power law scheme in analyzing velocity profiles.

3. Extension of the study to include laminar flow fields. 


\section{REFERENCES}

[1] Awuor KO. simulating Natural Turbulent Convection Fluid Flow in an Enclosure the Two-Equation Turbulent Models Ph.D. Thesis. UNIVERSITY NAME; 2013

[2] Kane, M., Cheikh, M., Lamine, S and Joseph, S. "Natural Convection of Air in a Square Cavity with Partially Thermally Active Side Walls". Open Journal of Fluid Dynamics, vol. 7, no.4

[3] Noor-A-Alam S. (2015). "Free Convection Heat Transfer in a Square Cavity heated from below and symmetrically cooling from the sides." International Journal of innovative Science Engineering and Technology, 2015; 2(8); 2348-79

[4] Wilcox DC. Turbulence modeling for CFD. La Canada, CA: DCW industries, 1998

[5] Menter FR. Two-equation eddy-viscosity turbulence models for engineering applications. AIAA Journal. 1994; 32 (8): 1598-1605.

[6] Versteeg HK, Malalasekera W. (2007). An introduction to computational fluid dynamics: the finite volume method. Pearson Education, 2007.

[7] Abdelkalder B, Karim R, Youb K. Numerical Study of Mixed Convection Heat Transfer in a Lid-Driven Cavity Filled with a nano fluid. Mechanics and Industry. 2015; 16: 505.

[8] Ampofo F, Karayiannis TG. Experimental benchmark data for turbulent natural convection in an air filled square cavity. International Journal of Heat and Mass Transfer. 2003; 46(19): 3551-3572.

[9] Anderson JD, Wendt J. Computational fluid dynamics. New York: McGraw-Hill, 1995.

[10] Dalal A, Das MK. Natural convection in a rectangular cavity heated from below and uniformly cooled from the top and both sides. Numerical Heat Transfer, Part A: Applications. 2006; 49(3): 301-322.

[11] de Vahl Davis G. Finite difference methods for natural and mixed convection in enclosures. International Heat Transfer Conference Digital Library. Begel House Inc, 1986.

[12] Edward M, Sigey J, Okello J, Okwoyo J. Natural Convection with Localized Heating and Cooling on Opposite Vertical Walls in an Enclosure. CNCE 1. 2013; 4: 72-78.

[13] Ferziger JH, Perić M, Street RL. Computational methods for fluid dynamics. Berlin: Springer, 2002.

[14] Gikundi A, Awuor K, Karanja S. A numerical study of Turbulen Natural Convection in a rectangular enclosure heated from below. German Journal of Advanced Scientific Research. 2020; 3(4).

[15] Jones WP, Launder BE. The prediction of laminarization with a two-equation model of turbulence. International Journal of Heat and Mass Transfer. $1972 ; 15(2): 301-314$.

[16] Karanja S, Sigey J, Gatheri F, Kirima, E. Turbulent Natural Convection in an Enclosure at Varying Aspect Ratio.

[17] Layton WR. The 1877 Boussinesq Conjecture: Turbulent Fluctuations Are Dissipative on the Mean Flow, 2014.

[18] Onyango O, Sigey J, Okelo AJ, Okwoyo J. (2013). Enhancement of natural convection heat transfer in a square enclosure with localized heating from below. International Journal of Science and Research. 2013; 2(10): 82-87.

[19] Oosthuizen P, Paul J. (2010). The effect of end conditions on laminar and turbulent natural convective flow in a high aspect ratio enclosure. 10th AIAA/ASME Joint Thermophysics and Heat Transfer Conference. 2010: 5066.

[20] Patankar SV. Numerical heat transfer and fluid flow. Washington, DC, Hemisphere Publishing Corp., 1980.

[21] Rodi W. Turbulence models and their application in hydraulics. CRC Press, 1993.

[22] Safaei M, Mohammadi M. (2011). Numerical modeling of turbulence mixed convection heat transfer in air filled enclosures by finite volume method. The International Journal of Multiphysics. 2011; 5(4): 307-324.

[23] Salih EA. Effects of aspect ratio on natural convection heat transfer in a parallelogrammic enclosure heated from below. ZANCO Journal of Pure and Applied Sciences. 2015; 27(4): 45-56.

[24] Sarris IE, Lekakis I, Vlachos NS. Natural convection in rectangular tanks heated locally from below. International Journal of Heat and Mass Transfer. 2004; 47(14-16): 3549-3563.

[25] Sharif ML, Mohammed LA. (2005). Unsteady free convection and mass transfer in rectangular cavities using the Finite Volume based computational method. Thermal International Journal. 2005; 10: 568-789.

[26] Tryggvason G. Chapter 6-Computational Fluid Dynamics. PK Kundu, IM Cohen, and DRBT-FM (Sixth E. Dowling, Eds. Boston, $227-291$. 